\title{
Comparison between conventional and compressed sensing cine cardiovascular magnetic resonance for feature tracking global circumferential strain assessment
}

Tomoyuki Kido ${ }^{1 *} \mathbb{0}$, Kuniaki Hirai ${ }^{2}$, Ryo Ogawa ${ }^{1}$, Yuki Tanabe ${ }^{1}$, Masashi Nakamura ${ }^{1}$, Naoto Kawaguchi ${ }^{1}$, Akira Kurata', Kouki Watanabe ${ }^{3}$, Michaela Schmidt ${ }^{4}$, Christoph Forman ${ }^{4}$, Teruhito Mochizuki ${ }^{5,6}$ and Teruhito Kido ${ }^{1}$

\begin{abstract}
Background: Feature tracking (FT) has become an established tool for cardiovascular magnetic resonance (CMR)based strain analysis. Recently, the compressed sensing (CS) technique has been applied to cine CMR, which has drastically reduced its acquisition time. However, the effects of CS imaging on FT strain analysis need to be carefully studied. This study aimed to investigate the use of CS cine CMR for FT strain analysis compared to conventional cine CMR.

Methods: Sixty-five patients with different left ventricular (LV) pathologies underwent both retrospective conventional cine CMR and prospective CS cine CMR using a prototype sequence with the comparable temporal and spatial resolution at 3 T. Eight short-axis cine images covering the entire LV were obtained and used for LV volume assessment and FT strain analysis. Prospective CS cine CMR data over 1.5 heartbeats were acquired to capture the complete end-diastolic data between the first and second heartbeats. LV volume assessment and FT strain analysis were performed using a dedicated software ( $\mathrm{ci}^{42}$; Circle Cardiovasacular Imaging, Calgary, Canada), and the global circumferential strain (GCS) and GCS rate were calculated from both cine CMR sequences.

Results: There were no significant differences in the GCS $(-17.1 \%[-11.7,-19.5]$ vs. $-16.1 \%[-11.9,-19.3$; $p=0.508)$ and GCS rate $(-0.8[-0.6,-1.0]$ vs. $-0.8[-0.7,-1.0] ; p=0.587)$ obtained using conventional and CS cine CMR. The GCS obtained using both methods showed excellent agreement $(y=0.99 x-0.24 ; r=0.95 ; p<0.001)$. The Bland-Altman analysis revealed that the mean difference in the GCS between the conventional and CS cine CMR was $0.1 \%$ with limits of agreement between $-2.8 \%$ and $3.0 \%$. No significant differences were found in all LV volume assessment between both types of cine CMR.
\end{abstract}

Conclusion: CS cine CMR could be used for GCS assessment by CMR-FT as well as conventional cine CMR. This finding further enhances the clinical utility of high-speed CS cine CMR imaging.

Keywords: Compressed sensing, Cardiovascular magnetic resonance, Feature tracking, Myocardial strain

*Correspondence: tomozo0421@gmail.com

1 Department of Radiology, Ehime University Graduate School of Medicine, Hitsukawa, Toon, Ehime 791-0295, Japan

Full list of author information is available at the end of the article

\section{Background}

Left ventricular (LV) wall motion assessment is important in the management of ischemic heart disease and cardiomyopathy [1]. Various imaging modalities, including original author(s) and the source, provide a link to the Creative Commons licence, and indicate if changes were made. The images or other third party material in this article are included in the article's Creative Commons licence, unless indicated otherwise in a credit line to the material. If material is not included in the article's Creative Commons licence and your intended use is not permitted by statutory regulation or exceeds the permitted use, you will need to obtain permission directly from the copyright holder. To view a copy of this licence, visit http://creativecommons.org/licenses/by/4.0/. The Creative Commons Public Domain Dedication waiver (http://creativeco mmons.org/publicdomain/zero/1.0/) applies to the data made available in this article, unless otherwise stated in a credit line to the data. 
echocardiography [2], scintigraphy [3], computed tomography $(\mathrm{CT})$ and cardiovascular magnetic resonance (CMR) [4] can be used for this purpose. Especially, retrospective electrocardiogram (ECG)-gated balanced steady-state free-precession (bSSFP) cine CMR can be used to assess the LV wall with high resolution and reproducibility [5-8]. Nonetheless, myocardial strain analyses using several advanced CMR techniques, including tagging [9], displacement encoding with stimulated echoes [10], strain encoding [11], and tissue phase mapping [12] have been shown to be superior to wall motion assessments for detecting differences in myocardial deformation. The global circumferential strain (GCS) assessment has been reported to be an independent prognostic indicator of both asymptomatic patients and those with heart failure $[13,14]$. However, although these techniques can provide reference standard measurements of myocardial strain, they require additional time-consuming sequences that are often clinically impractical.

Feature tracking (FT), an algorithm that was originally developed for echocardiographic images, was introduced as a technique for the analysis and quantification of myocardial strain based on bSSFP cine images $[15,16]$. This technique can also be applied to conventional cine CMR [17] to retrospectively analyze myocardial strain without additional acquisition of strain specific image sequence.

Recently, the compressed sensing (CS) technique with incoherent sampling and iterative reconstruction was suggested as a solution to accelerate CMR acquisition time [18-20]. The CS approach can drastically reduce the cine CMR scan time and improve patient compliance. In addition, previous studies demonstrated the utility of CS cine CMR for LV volume evaluation [21-23]. However, the effects of CS cine CMR imaging for FT strain analysis remains to be carefully studied. This study investigated the effects and adequacy of using CS cine CMR images for FT strain analysis compared to conventional cine CMR.

\section{Methods \\ Study population}

The present study was reviewed and approved by the ethics review board of Saiseikai Matsuyama Hospital, Matsuyama, Japan. Written informed consent was obtained from all participants. A total of 74 consecutive patients scheduled for CMR for various cardiac conditions were enrolled. All patients underwent CMR scanning including both conventional cine CMR and CS cine CMR sequences. Patients with arrhythmia $(n=6)$ and severely impaired breath-hold capacity $(n=3)$ were excluded from this investigation to avoid potentially poor cine CMR image quality. As a result, 65 patients were included in the analysis, and their characteristics are shown in Table 1.

\section{Cine CMR data acquisition}

All CMR examinations were performed at 3 T (MAGNETOM Skyra; Siemens Healthineers, Erlangen, Germany) with 18-channel body matrix coil and 32-channel spine matrix coil. After scout images were obtained to plan the cardiac axis views, eight short-axis cine CMR images covering the entire LV were obtained. The segmented bSSFP sequence was used for the retrospective ECG-gated conventional cine CMR scans. Immediately afterward, a prospective ECG-triggered CS cine CMR scanwas performed using a prototype sequence. The data acquisition of CS cine CMR is single-shot using incoherent sampling of $\mathrm{k}$-space. This is realized with a random distribution of the readouts on the Cartesian grid in k-space. Image reconstruction was performed with a non-linear, iterative SENSE-type approach implementing spatio-temporal regularization using redundant Haar wavelets [24]. The corresponding cost function was solved with a fast iterative shrinkage-threshold algorithm (FISTA) type optimization consisting of a gradient descend step for the quadratic terms and the evaluation of the proximal operator. The proximal operator is weighted with the regularization parameter, which was set to 0.001 and 0.005 for spatial and temporal regularization, respectively. Details regarding data acquisition and reconstruction of CS cine CMR have been described elsewhere [23]. We acquired the prospective CS cine CMR data for 1.5 heartbeats, definitely including the end-diastolic phase between the first and second heartbeat [23]. Both the temporal and spatial resolution, as well as the slice orientation, were kept identical between the two cine CMR

Table 1 Clinical characteristics of the study population

\begin{tabular}{ll}
\hline Number of patients & $\mathbf{6 5}$ \\
\hline Age (years) & $70.9 \pm 9.2$ \\
Gender (female/male) & $18 / 47$ \\
Height (cm) & $160.8 \pm 9.5$ \\
Weight $(\mathrm{kg})$ & $62.1 \pm 13.2$ \\
BMl $\left(\mathrm{kg} / \mathrm{m}^{2}\right)$ & $23.8 \pm 3.4$ \\
Heart rate (bpm) & $62 \pm 10$ \\
Clinical diagnosis & \\
Coronary artery disease & $44(68 \%)$ \\
Cardiomyopathy & $14(22 \%)$ \\
Valve disease & $3(5 \%)$ \\
Other & $4(6 \%)$ \\
\hline
\end{tabular}

Data are presented as $\mathrm{n}(\%)$ and mean \pm standard deviation, unless stated otherwise

$B M I$ body mass index 
protocols. In both conventional and CS cine scans, actual one cine phase was composed by $41.1 \mathrm{~ms}$ data acquisition ( $3.16 \mathrm{~ms}$ TR $\times 13$ segments). And the conventional cine image was interpolated to 25 cine phases by calculated phase using retrospective gating with 1 heartbeat. The calculate phase interpolation was not applied for CS cine image using prospective triggering with 1.5 heartbeat. Detailed imaging parameters are listed in Table 2.

\section{LV volume and strain assessment}

Conventional and CS cine CMR images were analyzed using commercially available software ( $\mathrm{cvi}^{42}$; Circle Cardiovascular Imaging, Calgary, Alberta, Canada) which has been previously validated [25]. LV volumes and mass were assessed by contouring the LV endocardial and epicardial borders in end-diastole and end-systole in both cine CMR images following the recommendations of Society for Cardiovascular Magnetic Resonance for post-processing [26]. The FT software algorithm could automatically draw both the endocardial and epicardial contours as well as track image features, including signal inhomogeneities, tissue patterns of the myocardium, and anatomic structures throughout the whole cardiac cycle. Additionally, the software allowed editing of the border when inadequate contours were reported (Fig. 1).

To investigate the effects of CS imaging for FT strain assessment, the GCS and GCS rate were calculated using the two cine CMR images. Specifically, the GCS was calculated as the peak circumferential strain, using the mean of the eight-slice cine CMR images (Fig. 2). Given that the GCS is a measure of circumferential shortening from the baseline, it is typically a negative value. Therefore, greater circumferential shortening is indicated by an increasingly negative value.

The intra- and inter-observer variability of the FT strain assessment were performed in 20 randomly selected subjects. To measure the intra-observer variability, an interval of 4 weeks was chosen between the first and second analysis. Two independent blinded radiologists were employed to investigate the inter-observer variability.

\section{Image contrast assessment}

To investigate the effects of using CS on image contrast, the contrast ratio (CR) between blood and myocardium was calculated in 20 randomly selected subjects. The regions of interest (minimum size: $80 \mathrm{~mm}^{2}$ ) were placed in both the midventricular septum and the LV lumen in an end-diastolic phase. The mean signal of the LV lumen was divided by that of the midventricular septum for $C R$ measurement.

\section{Statistical analysis}

Statistical analyses were performed using the commercially available software (JMP version 11; SAS Institute, Cary, North Carolina, USA). Continuous data are expressed as the mean \pm standard deviation (SD) or the median (first quartile, third quartile) depending on the distribution. The Wilcoxon signed-rank test was used to compare the LV volume, the GCS and GCS rates obtained using the two cine CMR. The Spearman rank correlation

Table 2 CMR sequence parameters

\begin{tabular}{lll}
\hline & Conventional cine & CS cine \\
\hline Sequence type & 2 D cine TrueFISP & 2 cine TrueFISP \\
ECG mode & Retrospective gating & Prospective triggering \\
Repetition time (ms) & 3.16 & 3.16 \\
Echo time (ms) & 1.4 & 1.4 \\
FOV (mm ${ }^{2}$ ) & $350 \times 350$ & $350 \times 350$ \\
Image matrix & $208 \times 166$ & $208 \times 166$ \\
Spatial resolution reconstructed $\left(\mathrm{mm}^{2}\right)$ & $1.7 \times 1.7$ & $1.7 \times 1.7$ \\
Temporal resolution (ms) (repetition time $\times$ segments) & 41.1 & 41.1 \\
Slice thickness (mm) & 6 & 6 \\
Slice numbers & 8 & 8 \\
Flip angle (degrees) & 50 & 50 \\
Bandwidth (Hz/pixel) & 1145 & 1145 \\
Number of segments & 13 & 13 \\
Cardiac phases & 25 & $19-31$ \\
Breath-holds (n) & 4 & 1 \\
Acceleration factor & 3 & 12.8
\end{tabular}

CS compressed sensing, ECG electrocardiogram, FOV field of view 
a

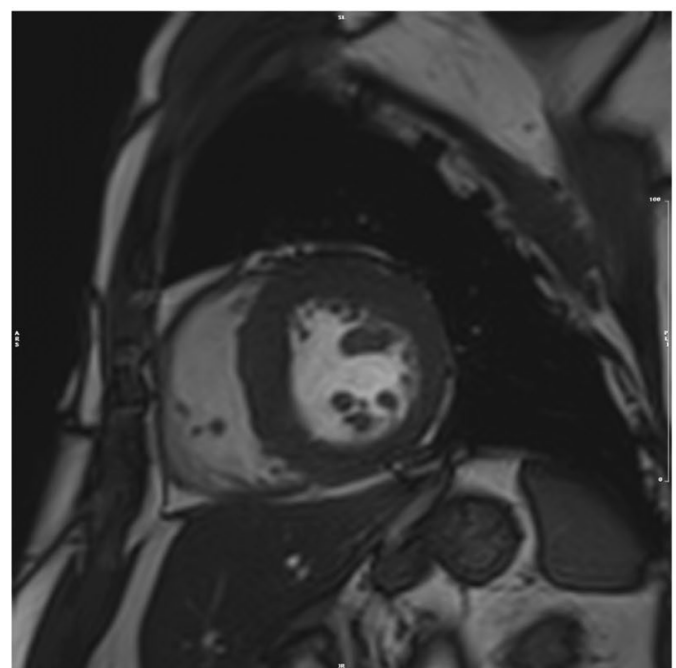

b

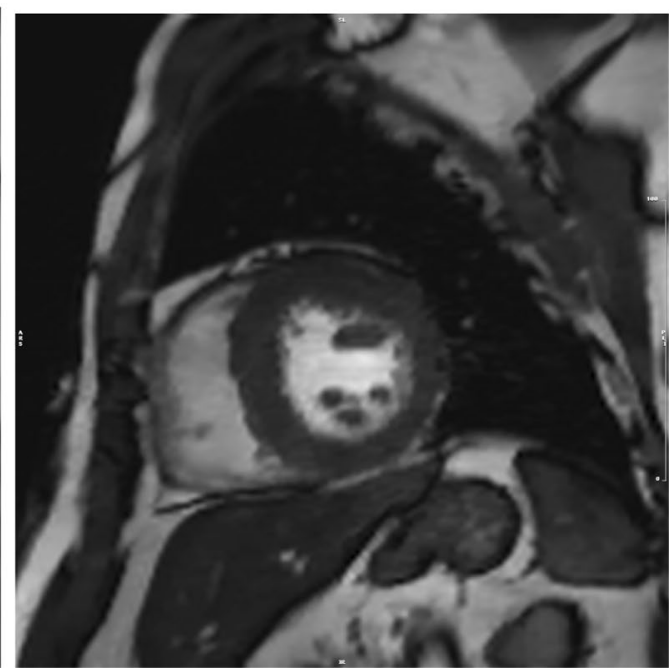

Fig. 1 End-diastolic short-axis images obtained using conventional cine cardiovascular magnetic resonance (CMR) (a) and compressed sensing (CS) cine CMR (b)

coefficient and Bland-Altman analysis [27] were used to evaluate the correlation and agreement. Furthermore, intra-observer and inter-observer variability were evaluated by performing Bland-Altman analysis and calculating the intraclass correlation coefficient (ICC). The blood-myocardium CR determined by both cine CMR methods were compared using the paired $t$ test. A $p$ value $<0.05$ was considered statistically significant.

\section{Results}

All 65 patients successfully underwent scans using both conventional and CS cine CMR sequences. Furthermore, all conventional and CS cine CMR images were eligible for LV volume assessments and CMR-FT analyses. The total examination time was $113 \pm 7 \mathrm{~s}$ and $24 \pm 4 \mathrm{~s}$ for conventional and CS cine CMR, respectively $(\mathrm{p}<0.001)$. For all LV volume measurements, no significant differences were found between conventional and CS cine CMR (Table 3).

There were no significant differences in GCS and GCS rate between conventional and CS cine CMR (Table 4). Furthermore, the GCS obtained using both methods showed good agreement according to the linear regression analysis $(y=0.99 x-0.24 ; \mathrm{r}=0.95 ; \mathrm{p}<0.001)$. Additionally, the Bland-Altman analysis revealed that the mean difference between conventional and CS cine CMR was $0.1 \%$ with limits of agreement between $-2.8 \%$ and $3.0 \%$ (Fig. 3).

Similarly, the intra- and inter-observer variability yielded a good agreement in both the conventional and CS cine CMR (Fig. 4), and their ICC were excellent (conventional cine CMR: ICC $=0.99$ and 0.98 for intraand inter-observer variability, respectively; CS cine CMR: ICC $=0.99$ and 0.98 for intra- and inter-observer, respectively).

Finally, the blood-myocardium CR did not differ significantly between the two methods (conventional cine CMR: 5.0 \pm 0.6 ; CS cine CMR: $4.9 \pm 0.7 ; \mathrm{p}=0.956$ ).

\section{Discussion}

The strain assessment based on myocardial tagging is the most established technique utilized for measuring myocardial strain $[13,14]$. However, the acquisition of additional images and the necessity of post-processing limits its use in routine clinical practice. Since its introduction [15], CMR-FT has been widely used for strain assessment in both clinical and research settings [16, 17, 28, 29]. CMR-FT can be applied to routine cine CMR; therefore, it has the potential to retrospectively evaluate myocardial strain in patients who did not undergo specific strain imaging.

The CS technique has been used for cardiovascular cine CMR. A previous study suggested using it to drastically shorten the imaging time while maintaining accurate LV volume quantification [23]. Because the widespread clinical use of CS cine CMR is desirable, it is essential to show that FT strain assessment results obtained using CS cine CMR are reproducible and comparable to those obtained using conventional cine CMR. The present study demonstrated that CS cine CMR could be used to assess GCS with high reproducibility as well as conventional cine CMR. This finding suggests that we can perform FT 


\section{a}

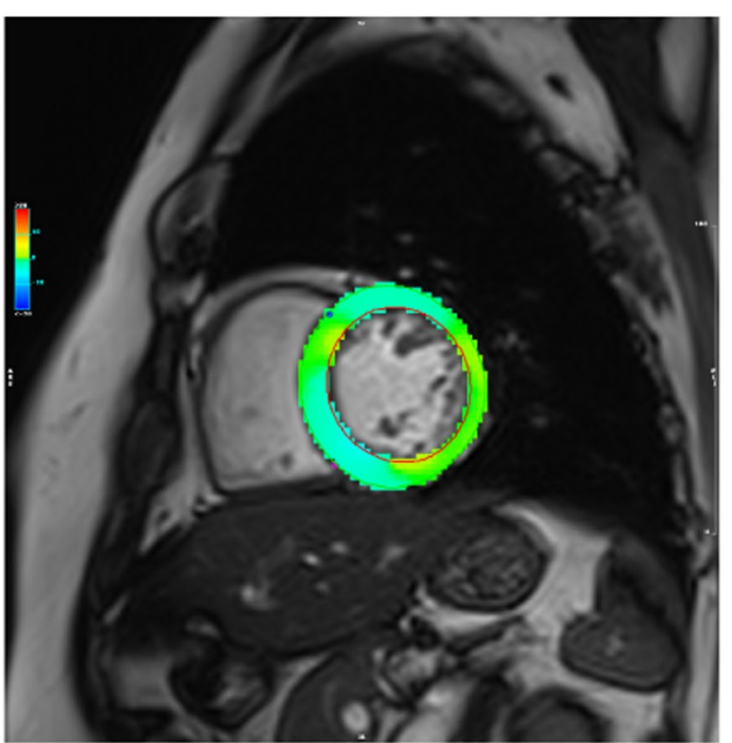

C

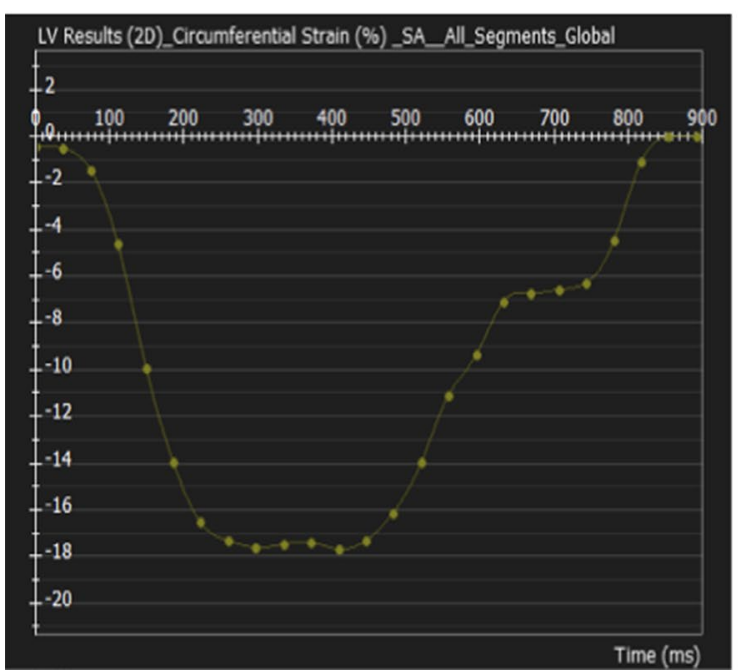

b

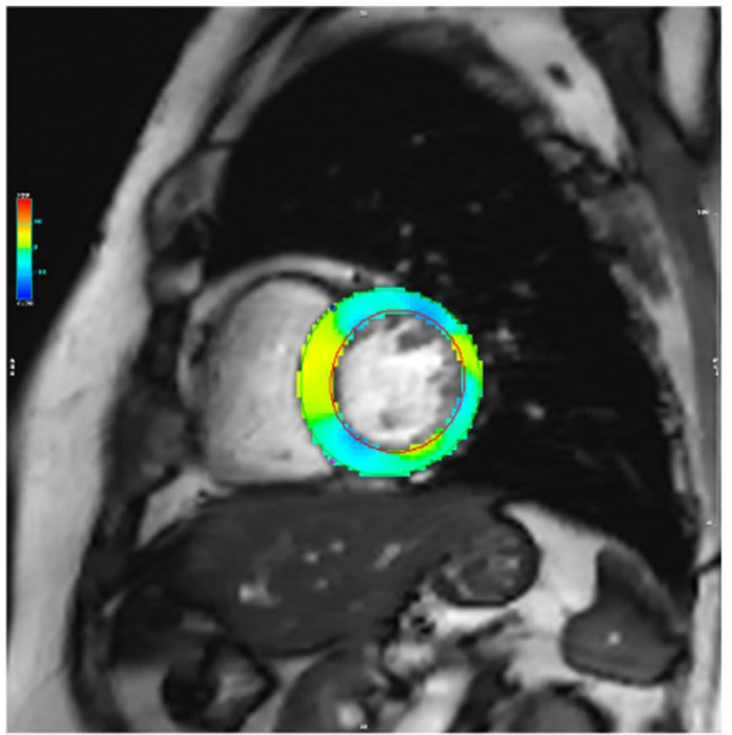

d

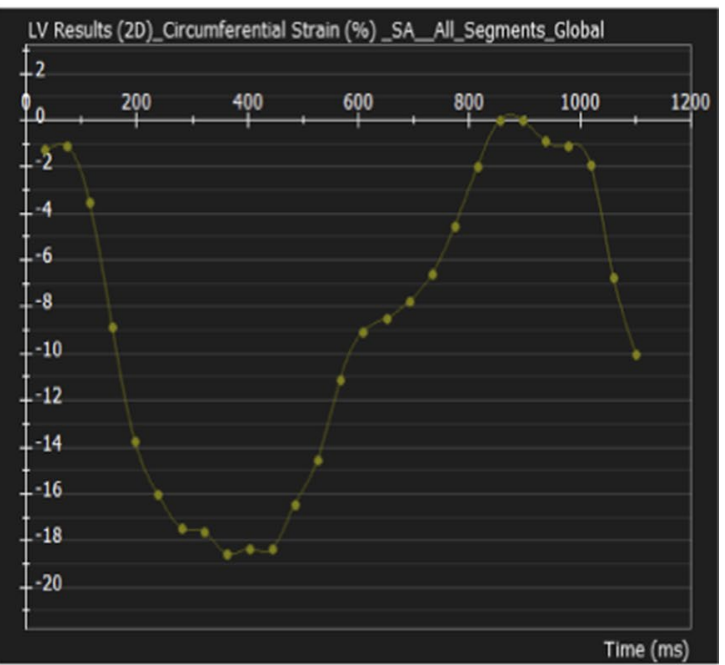

Fig. 2 Example of strain assessment by CMR feature tracking (CMR-FT) in conventional (a, $\mathbf{c})$ and CS cine CMR (b, d). The curves in the graph represent the similar global circumferential strains

Table 3 Left Ventricular volume measurements with Conventional and CS cine CMR

\begin{tabular}{lllll}
\hline & Conventional cine $(\mathbf{n}=\mathbf{6 5})$ & CS cine $(\mathbf{n}=\mathbf{6 5})$ & Mean difference & P value \\
\hline EDV $(\mathrm{ml})$ & $112.1[95.7,140.0]$ & $111.4[95.6,154.9]$ & $0.3 \pm 8.3$ & 0.84 \\
ESV $(\mathrm{ml})$ & $47.2[33.5,78.6]$ & $51.0[34.3,79.1]$ & $-0.3 \pm 6.4$ & 0.36 \\
SV $(\mathrm{ml})$ & $65.4[52.8,73.9]$ & $62.1[48.9,76.4]$ & $0.6 \pm 9.8$ & 0.70 \\
Mass $(\mathrm{g})$ & $92.6[75.8,120.3]$ & $92.2[75.1,117.3]$ & $0.9 \pm 5.9$ & 0.29 \\
EF $(\%)$ & $58.6[46.4,66.3]$ & $57.2[46.2,64.4]$ & $1.1 \pm 5.4$ & 0.09 \\
\hline
\end{tabular}

Data are presented as the medians [first quartile, third quartile]

Mean differences are presented as mean \pm standard deviation

$E D V$ end-diastolic volume, EF ejection fraction, ESV end-systolic volume, SV stroke volume 
Table 4 Results of GCS and GCS rate from conventional and CS cine CMR

\begin{tabular}{llll}
\hline & $\begin{array}{l}\text { Conventional cine } \\
(\mathbf{n}=\mathbf{6 5})\end{array}$ & CS cine $(\mathbf{n}=\mathbf{6 5})$ & $\mathbf{p}$ \\
\hline GCS $(\%)$ & $-16.1[-11.9,-19.3]$ & $-17.1[-11.7,-19.5]$ & 0.508 \\
GCS rate $\left(\mathrm{s}^{-1}\right)$ & $-0.8[-0.6,-1.0]$ & $-0.8[-0.7,-1.0]$ & 0.587 \\
\hline
\end{tabular}

Data are presented as the median [first quartile, third quartile]

CS compressed sensing, GCS global circumferential strain
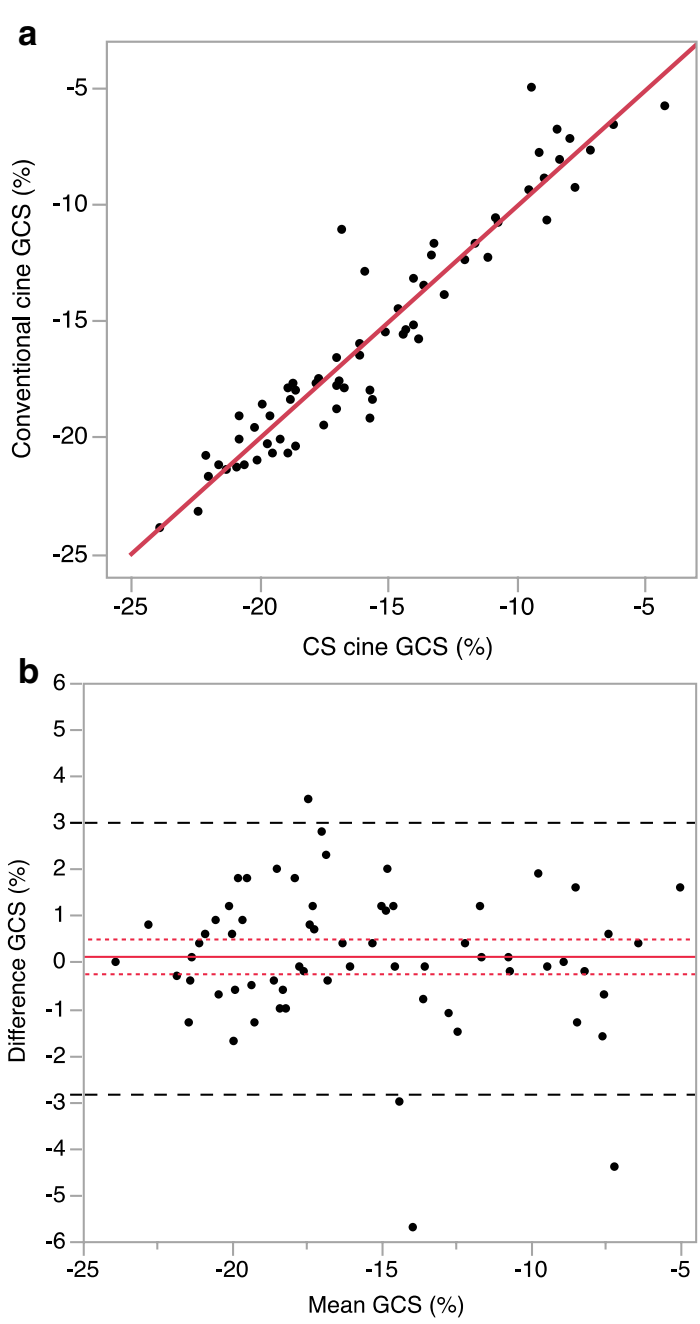

Fig. 3 Scatter plots (a) and Bland-Altman plots (b) of global circumferential strain (GCS) measurements by CMR-FT. The solid line indicates the difference between the two sequences. The long-dashed lines represent the $95 \%$ agreement interval (i.e., the mean \pm 1.96 SD). The short-dashed lines indicate the $95 \%$ confidence interval of the mean difference

strain assessments using CS cine CMR and compare the results with those previously obtained using conventional cine CMR. The ability of CS cine CMR to assess both LV volume and strain within a short imaging time enhances its clinical utility.

Regarding the image features, the CS cine CMR images are associated with reduced noise but with a slightly pixelated and textured appearance compared with conventional cine CMR images due to its noise reduction process during the reconstruction step. Although the image features of CS cine CMR were slightly different from those of conventional cine CMR, good bloodmyocardium $\mathrm{CR}$ was maintained and no significant differences were observed. Because that the FT algorithm is highly dependent on high contrast between the blood and myocardium [30, 31], similar results could be obtained during FT strain analysis.

Langton et al. evaluated FT strain based on images acquired with the CS real-time sequence that was prospectively triggered and acquired data for one heartbeat [32]. They showed there were highly consistent with conventional cine, but small systematic differences were observed. The prospective ECG-triggered cine CMR scan is useful, but capturing the complete end-diastolic data with this technique remains difficult because a finite time is required to detect the next ECG trigger [21, 22]. Because the strain value is indicated by the rate of change from the end-diastole (i.e., the reference point), the GCS may be underestimated using a one-heartbeat prospective cine CMR scan (i.e., the reference end-diastolic data may be missing). Therefore, it is crucial to acquire data of the whole cardiac cycle to perform accurate LV volume assessment and FT strain analysis using prospective cine CMR. In our study, prospective CS cine CMR data for 1.5 heartbeats were obtained to overcome this limitation and to capture the complete end-diastolic data between the first and second heartbeats.

We acquired CS cine CMR images using the comparable temporal and spatial resolution as conventional cine CMR in the current study; however, the effects of the differences in spatial resolution on FT have been reported [33]. Moreover, if the temporal resolution is too low, then it is expected that the strain value will be underestimated without capturing the complete diastole and systole phase. Furthermore, during CS cine CMR image reconstruction, the regularization setting also seems to affect the image quality and FT strain assessment results.

In our LV volume assessment, there was no significant difference, but CS cine tended to slightly overestimate end-systolic volume, probably as a result of the temporal regularization. Langton et al. found that good agreement to a conventional cine for GCS as in our study, but slightly less agreement for global radial strain ${ }^{32}$. Since radial strain reflects changes in myocardial wall thickness and is highly nonhomogeneous (increasing toward the endocardium), it is likely more sensitive to small differences 
a

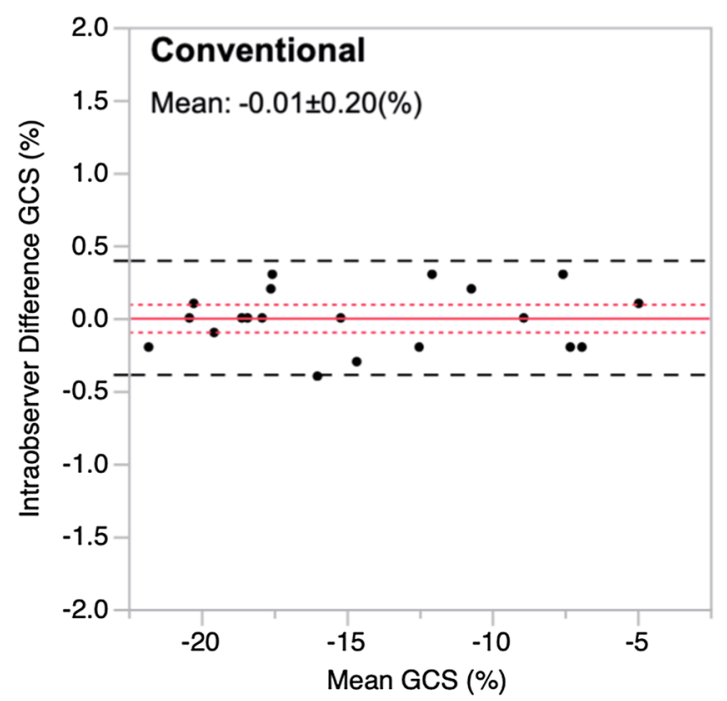

c

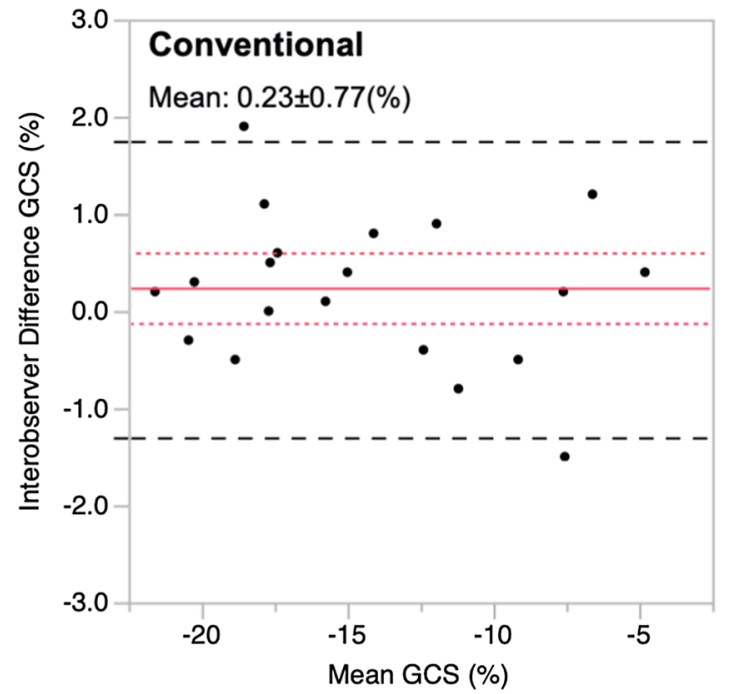

b

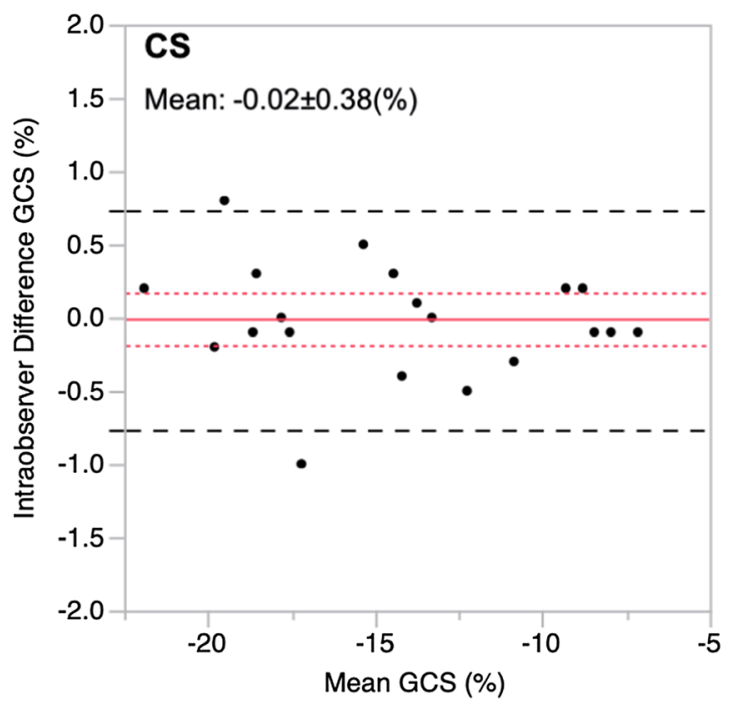

d

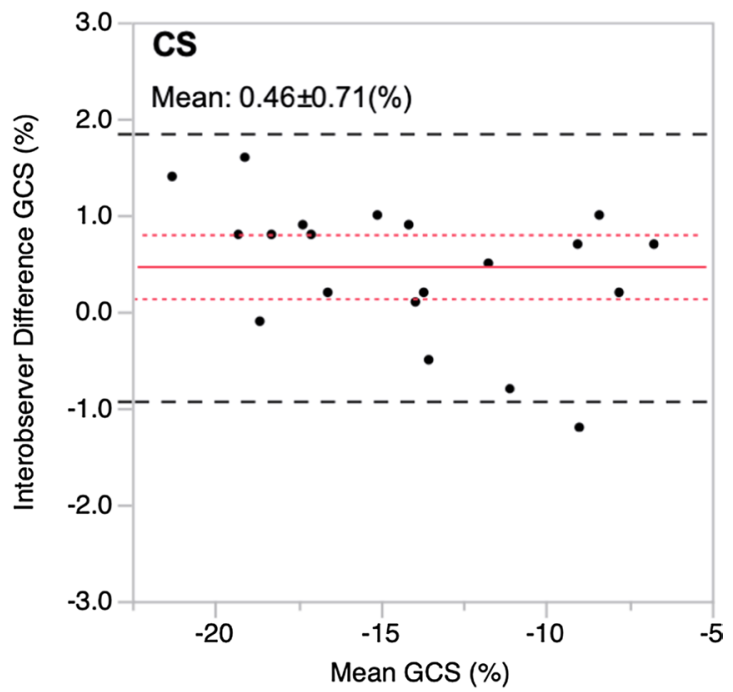

Fig. 4 Bland-Altman plots of intra-observer variability of conventional (a) and CS (b) cine CMR. Bland-Altman plots of inter-observer variability of conventional (c) and CS (d) cine CMR. The solid line indicates the difference between the two sequences. The long-dashed lines represent the 95\% agreement interval (i.e., the mean \pm 1.96 SD). The short-dashed lines indicate the $95 \%$ confidence interval of the mean difference

in myocardial contouring. In our experience, too high regularization is likely to blur and smooth CS cine CMR images in spatial domain and to be insensitive with small motion in temporal domain. It would lead to an underestimation of strain. In this study, we used the fixed regularization value ( 0.001 for spatial regularization and 0.005 for temporal regularization), which was set based on the image quality of CS cine CMR, and this setting helped to maintaining the features necessary to calculate strain. We did not investigate the effects of changes in regularization during the FT strain analysis. Therefore, further detailed examinations of CS parameters are required and we should still be cautious standardized image acquisition to make FT strain measurements reliable and comparable.

\section{Limitations}

There were some limitations to the present study. First, that the effects of using different types of CMR-FT analysis software were not assessed. Since the algorithm differs depending on the software, these inherent differences should be considered when comparing results obtained using different software [25]. Second, we did 
not obtain long-axis views using the CS cine CMR for global longitudinal strain (GLS) assessment, which may represent a strong predictor of outcomes and may potentially elucidate subtle changes in myocardial function $[34,35]$. Further evaluations including GLS assessments using long-axis CS cine CMR are warranted to clarify the usefulness of CS cine CMR imaging for FT strain assessment.

\section{Conclusion}

CS cine CMR images could be used for FT strain assessment with similar high reproducibility as conventional cine CMR. The ability of CS cine CMR to assess both LV volume and FT strain within a short imaging time enhances its clinical utility.

\section{Abbreviations}

bSSFP: Balanced steady state free precession; Cl: Confidence interval; CMR: Cardiovascular magnetic resonance; CR: Contrast ratio; CS: Compressed sensing; CT: Computed tomography; ECG: Electrocardiogram; EDV: End-diastolic volume; EF: Ejection fraction; ESV: End-systolic volume; FISTA: Fast iterative shrinkage-threshold algorithm; FT: Feature tracking; GCS: Global circumferential strain; GLS: Global longitudinal strain (GLS); LV: Left ventricle/left ventricular; SD: Standard deviation; SV: Stroke volume.

\section{Acknowledgements}

The authors are grateful to Yoshiaki Komori of Siemens Healthcare K.K. (Tokyo, Japan) for the optimization of the sequence parameters and image quality in the present study.

\begin{abstract}
Authors' contributions
TK conceived the study, quantitatively measured the strain and image contrast, performed the statistical analysis, and drafted the manuscript. $\mathrm{KH}$ quantitatively measured the strain. KW participated in the design of the study and its coordination. CF and MS contributed to the sequence development and the implementation on the scanner, as well as the revision of the manuscript. RO, YT, MN, NK, AK, TM and TK assisted with the interpretation of the results and helped revise the manuscript. All authors read and approved the final manuscript.
\end{abstract}

\section{Funding}

Not applicable.

\section{Availability of data and materials \\ Not applicable.}

\section{Ethics approval and consent to participate}

The study protocol was approved by the Ethical Review Board of the Saiseikai Matsuyama Hospital, and written informed consent was obtained from all participants.

\section{Consent for publication}

All the participants in this study provided written consent for publication.

\section{Competing interests}

The authors declare that they have no competing interests.

\section{Author details}

${ }_{1}^{1}$ Department of Radiology, Ehime University Graduate School of Medicine, Hitsukawa, Toon, Ehime 791-0295, Japan. ${ }^{2}$ Department of Radiology, Uwajima City Hospital, Uwajima, Japan. ${ }^{3}$ Department of Cardiology, Saiseikai Matsuyama Hospital, Matsuyama, Japan. ${ }^{4}$ Siemens Healthcare $\mathrm{GmbH}$, Erlangen, Germany. ${ }^{5}$ Department of Radiology, Yoshino Hospital, Imabari,
Japan. ${ }^{6}$ Department of Radiology, I.M. Sechenov First Moscow State Medical University, Moscow, Russia.

Received: 3 July 2020 Accepted: 6 January 2021

Published online: 22 February 2021

\section{References}

1. Cicala S, de Simone G, Roman MJ, Best LG, Lee ET, Wang W, et al. Prevalence and prognostic significance of wall-motion abnormalities in adults without clinically recognized cardiovascular disease: the strong heart study. Circulation. 2007;1 16:143-50.

2. Miyatake K, Yamagishi M, Tanaka N, Uematsu M, Yamazaki N, Mine Y, et al. New method for evaluating left ventricular wall motion by color-coded tissue Doppler imaging: in vitro and in vivo studies. J Am Coll Cardiol. 1995;25:717-24.

3. Vaduganathan P, He ZX, Vick GW III, Mahmarian JJ, Verani MS. Evaluation of left ventricular wall motion, volumes, and ejection fraction by gated myocardial tomography with technetium 99 m-labeled tetrofosmin: a comparison with cine magnetic resonance imaging. J Nucl Cardiol. 1999:6:3-10.

4. Gotte MJ, Germans T, Russel IK, Zwanenburg JJM, Marcus JT, van Rossum $A C$, et al. Myocardial strain and torsion quantified by cardiovascular magnetic resonance tissue tagging: studies in normal and impaired left ventricular function. J Am Coll Cardiol. 2006;48:2002-11.

5. Rathi VK, Biedermann RW. Imaging of ventricular function by cardiovascular magnetic resonance. Curr Cardiol Rep. 2004;6:55-61.

6. Alfakih K, Plein S, Thiele H, Jones T, Ridgway JP, Sivananthanet MU. Norma human left and right ventricular dimensions for MRI as assessed by turbo gradient echo and steady-state free precession imaging sequences. J Magn Reson Imaging. 2003;17:323-9.

7. Moon JC, Lorenz CH, Francis JM, Smith GC, Pennellet DJ. Breath-hold FLASH and FISP cardiovascular MR imaging: left ventricular volume differences and reproducibility. Radiology. 2002;223:789-97.

8. Grothues F, Smith GC, Moon JC, Bellenger NG, Collins P, Klein HU, et al. Comparison of interstudy reproducibility of cardiovascular magnetic resonance with two-dimensional echocardiography in normal subjects and in patients with heart failure or left ventricular hypertrophy. Am J Cardiol. 2002:90:29-34.

9. Ibrahim ESH. Myocardial tagging by cardiovascular magnetic resonance: evolution of techniques-pulse sequences, analysis algorithms, and applications. J Cardiovasc Magn Reson. 2011;13:36.

10. Zhong X, Spottiswoode BS, Meyer CH, Kramer CM, Epstein FH. Imaging three-dimensional myocardial mechanics using navigator-gated volumetric spiral cine DENSE MRI. Magn Reson Med. 2010;64:1089-97.

11. Osman NF, Sampath S, Atalar E, Prince JL. Imaging longitudinal cardiac strain on short-axis images using strain-encoded MRI. Magn Reson Med. 2001;46:324-34.

12. Simpson R, Keegan J, Firmin D. Efficient and reproducible high resolution spiral myocardial phase velocity mapping of the entire cardiac cycle. J Cardiovasc Magn Reson. 2013;15:34.

13. Choi EY, Rosen BD, Fernandes VR, Yan RT, Yoneyama K, Donekal S, et al. Prognostic value of myocardial circumferential strain for incident heart failure and cardiovascular events in asymptomatic individuals: the multiethnic study of atherosclerosis. Eur Heart J. 2013;34:2354-61.

14. Cho GY, Marwick TH, Kim HS, Kim MK, Hong KS, Oh DJ. Global 2-dimensional strain as a new prognosticator in patients with heart failure. J Am Coll Cardiol. 2009;54:618-24.

15. Maret E, Todt T, Brudin L, Nylander E, Swahn E, Ohlsson JL, et al. Functional measurements based on feature tracking of cine magnetic resonance images identify left ventricular segments with myocardial scar. Cardiovasc Ultrasound. 2009;7:53.

16. Schuster A, Hor KN, Kowallick JT, Beerbaum P, Kuttyet S. Cardiovascular magnetic resonance myocardial feature tracking. Concepts and clinical applications. Circ Cardiovasc Imaging. 2016;9(4):e0004077.

17. Hor KN, Gottliebson WM, Carson C, Wash E, Cnota J, Fleck R, et al. Comparison of magnetic resonance feature tracking for strain calculation with harmonic phase imaging analysis. JACC Cardiovasc Imaging. 2010:3:144-51. 
18. Candes EJ, Wakin MB. An introduction to compressive sampling. IEEE Signal Process Mag. 2008;25:21-30.

19. Lustig M, Donoho D, Pauly JM. Sparse MRI: the application of compressed sensing for rapid MR imaging. Magn Reson Med. 2007;58:1182-95.

20. Gamper U, Boesiger P, Kozerke S. Compressed sensing in dynamic MRI. Magn Reson Med. 2008;59:365-73.

21. Vincenti G, Monney P, Chaptinel J, Rutz T, Coppo S, Zenge MO, et al. Compressed sensing single-breath-hold CMR for fast quantification of LV function, volumes, and mass. JACC Cardiovasc Imaging. 2014;7:882-92.

22. Feng L, Srichai MB, Lim RP, Harrison A, King W, Adluru G, et al. Highly accelerated real-time cardiac cine MRI using k-t SPARSE-SENSE. Magn Reson Med. 2013;70:64-74.

23. Kido T, Kido T, Nakamura M, Watanabe K, Schmidt M, Forman C, et al. Compressed sensing real-time cine cardiovascular magnetic resonance: accurate assessment of left ventricular function in a single-breath-hold. J Cardiovasc Magn Reson. 2016;18(1):50.

24. Liu J, Rapin J, Chang T, Lefebvre A, Zenge M, Mueller E, et al. Dynamic cardiac MRI reconstruction with weighted redundant Haar wavelets. ISMRM. 2012;20:178.

25. Schuster A, Stahnke VC, Unterberg-Buchwald C, Kowallick JT, Lamata P, Steinmetz $M$, et al. Cardiovascular magnetic resonance feature tracking assessment of myocardial mechanics: Intervendor agreement and considerations regarding reproducibility. Clin Radiol. 2015;70:989-98.

26. Schulz-Menger J, Bluemke DA, Bremerich J, Flamm SD, Fogel MA, Friedrich MG, et al. Standardized image interpretation and post processing in cardiovascular magnetic resonance: Society for Cardiovascular Magnetic Resonance (SCMR) Board of Trustees Task Force on Standardized Post Processing. J Cardiovasc Magn Reson. 2013;15:35.

27. Bland JM, Altman DG. Statistical methods for assessing agreement between two methods of clinical measurement. Lancet. 1986;1:307-10.

28. Schuster A, Paul M, Bettencourt N, Morton G, Chiribiri A, Ishida M, et al. Cardiovascular magnetic resonance myocardial feature tracking for quantitative viability assessment in ischemic cardiomyopathy. Int J Cardiol. 2013;166:413-20.

29. Kowallick JT, Kutty S, Edelmann F, Chiribiri A, Villa A, Steinmetz M, et al. Quantification of left atrial strain and strain rate using cardiovascular magnetic resonance myocardial feature tracking: a feasibility study. J Cardiovasc Magn Reson. 2014;16:60

30. Andre F, Steen H, Matheis P, Westkott M, Breuninger $K$, Sander $Y$, et al. Age- and gender-related normal left ventricular deformation assessed by cardiovascular magnetic resonance feature tracking. J Cardiovasc Magn Reson. 2015;17:25.

31. Kuetting DLR, Dabir D, Homsi R, Sprinkart AM, Luetkens J, Schild HH, et al. The effects of extracellular contrast agent (Gadobutrol) on the precision and reproducibility of cardiovascular magnetic resonance feature tracking. J Cardiovasc Magn Reson. 2016;18:30.

32. Langton JN, Lam HI, Cowan BR, Occleshaw CJ, Gabriel R, Lowe B, et al. Estimation of myocardial strain from non-rigid registration and highly accelerated cine CMR. Int J Cardiovasc Imaging. 2017;33:101-7.

33. Knobelsdorff-Brenkenhoff F, Schunke T, Reiter S, Scheck R, Höfling B, Pilz G. Influence of contrast agent and spatial resolution on myocardial strain results using feature tracking MRI. Eur Radiol. 2020. https://doi. org/10.1007/s00330-020-06971-x.

34. Stanton T, Leano R, Marwick TH. Prediction of all-cause mortality from global longitudinal speckle strain: comparison with ejection fraction and wall motion scoring. Circ Cardiovasc Imaging. 2009;2:356-64.

35. Kraigher-Krainer E, Shah AM, Gupta DK, Santos A, Claggett B, Pieske B, et al. Impaired systolic function by strain imaging in heart failure with preserved ejection fraction. J Am Coll Cardiol. 2014;63:447-56.

\section{Publisher's Note}

Springer Nature remains neutral with regard to jurisdictional claims in published maps and institutional affiliations.
Ready to submit your research? Choose BMC and benefit from:

- fast, convenient online submission

- thorough peer review by experienced researchers in your field

- rapid publication on acceptance

- support for research data, including large and complex data types

- gold Open Access which fosters wider collaboration and increased citations

- maximum visibility for your research: over $100 \mathrm{M}$ website views per year

At BMC, research is always in progress.

Learn more biomedcentral.com/submissions 